\title{
SEMICONDUCTOR FERROMAGNETIC STRUCTURES
}

\author{
T. STORY \\ Institute of Physics, Polish Academy of Sciences \\ Al. Lotników 32/46, 02-668 Warsaw, Poland
}

\begin{abstract}
Ferromagnetic semiconductor structures such as superlattices or trilayers form a new class of magnetic systems composed entirely of semiconductor materials. The examples are $\mathrm{Ga}_{1-x} \mathrm{Mn}_{x} \mathrm{As}-\mathrm{AlGaAs}$ with the ferromagnetic layer of GaMnAs semimagnetic (diluted magnetic) semiconductor or EuS-PbS with the ferromagnetic member (EuS) of the family of europium chalcogenides. We discuss the spectrum of perspective ferromagnetic semiconductor materials, the effect of size and stress on magnetic properties of ultrathin semiconductor ferromagnetic layers, and the effect of interlayer exchange in all-semiconductor systems.
\end{abstract}

PACS numbers: 75.50.Pp, 75.70.Cn

\section{Introduction - ferromagnetic semiconductors}

Recently, the impressive progress has been achieved in the field of low dimensional layered magnetic structures composed of ferromagnetic and nonmagnetic metals such as, e.g., Co-Cu multilayers [1-3]. The discovery of a variety of new effects such as, e.g., interlayer exchange coupling or giant magnetoresistance, has led to successful applications in magnetoresistive and magnetooptical sensors $[4,5]$. In order to extend this field to other (nonmetallic) systems, a number of attempts have been undertaken to grow hybrid systems built of ferromagnetic metals and semiconductors, e.g., $\mathrm{Fe}-\mathrm{Si}$ superlattices or $\mathrm{ZnSe}-\mathrm{Fe}$ and $\mathrm{GaAs}-\mathrm{Fe}$ heterostructures $[1,6]$. However, these efforts face the severe problem of poor epitaxial compatibility of ferromagnetic metals and elemental and compound semiconductors. In this work, we discuss a new approach to this problem based on the use of ferromagnetic-nonmagnetic structures built of semiconducting materials only.

Ferromagnetic properties are usually observed in metallic materials. The list of semiconducting or insulating ferromagnets is rather short (see monographs [7] and [8] on magnetic semiconductors). Ferromagnetic semiconductors can be divided into two groups: magnetic compounds such as $\mathrm{EuO}$ or $\mathrm{CuCr}_{2} \mathrm{Se}_{4}$, and diluted magnetic alloys (semimagnetic semiconductors) like $\mathrm{Sn}_{1-x} \mathrm{Mn}_{x} \mathrm{Te}$ or $\mathrm{Ga}_{1-x} \mathrm{Mn}_{x} \mathrm{As}$. 
The best known group of magnetic semiconductors is the family of Eu chalcogenides: $\operatorname{EuA}(\mathrm{A}=\mathrm{O}, \mathrm{S}, \mathrm{Se}$, or Te). From a magnetic point of view, EuA represents the system of localized magnetic moments of $\mathrm{Eu}^{2+}$ ions (electron configuration $4 f^{7}$ ) coupled by short-range superexchange interactions well described by an isotropic Heisenberg Hamiltonian. The magnetic order observed in EuA depends on lattice parameter (varying for different anions): EuTe is an antiferromagnet, EuSe shows both antiferromagnetic and ferromagnetic order depending on the temperature range and applied magnetic field, and both EuS and EuO are ferromagnets with Curie temperature $T_{\mathrm{C}}=16.6 \mathrm{~K}$ and $T_{\mathrm{C}}=69 \mathrm{~K}$, respectively [7-9]. Stoichiometric $\mathrm{EuO}$ and $\mathrm{EuS}$ are semi-insulating materials. These materials can also be doped, e.g., with $\mathrm{Gd}^{3+}$ ions or by controlling the deviations from stoichiometric composition [7-9]. The absorption edge of europium chalcogenides is located about $2 \mathrm{eV}$.

In the group of IV-VI semimagnetic semiconductors, ferromagnetic properties are observed in $\mathrm{Sn}_{1-x} \mathrm{Mn}_{x} \mathrm{Te}, \mathrm{Ge}_{1-x} \mathrm{Mn}_{x} \mathrm{Te}, \mathrm{Pb}_{1-x-y} \mathrm{Sn}_{y} \mathrm{Mn}_{x} \mathrm{Te}$ $(0.4 \leq y \leq 1)$ and $\mathrm{Pb}_{1-x-y} \mathrm{Ge}_{y} \mathrm{Mn}_{x} \mathrm{Te}(0.9 \leq y \leq 1)$ crystals. Due to the limited solubility of Mn magnetic ions in IV-VI matrices $(x \leq 0.1)$, the ferromagnetic transition in these materials is typically below $T_{\mathrm{C}} \leq 30 \mathrm{~K}$. The ferromagnetic exchange interactions in IV-VI semimagnetic semiconductors originate from the Ruderman-Kittel-Kasuya-Yoshida (RKKY) mechanism [10-13]. In the RKKY mechanism the interspin coupling is brought about by the spin polarization of conducting carriers interacting via the $s p-d$ exchange with the localized magnetic moments of magnetic ions. The RKKY interaction is particularly important in systems with high carrier concentration. Although in IV-VI materials Mn is electrically neutral, the required very high concentration of carriers is generated by native defects (metal vacancies) and can be controlled by changing the deviation from stoichiometry during the process of isothermal annealing. This method is very efficient in IV-VI materials and, e.g., in SnMnTe and GeMnTe the conducting hole concentration can be set in the range $p=10^{20}-10^{21} \mathrm{~cm}^{-3}$ independently of $\mathrm{Mn}$ concentration. This unique possibility resulted in the discovery of the effect of carrier concentration induced ferromagnetism in $\mathrm{PbSnMnTe}$, which showed that the ferromagnetic order is observed only for crystals with hole concentration higher than a threshold value of $p \approx 3 \times 10^{20} \mathrm{~cm}^{-3}$ [10]. Although in II-VI and III-V semimagnetic semiconductors the details of the RKKY mechanism are somewhat different, the requirement for carrier concentration of the order of $p \approx 10^{20} \mathrm{~cm}^{-3}$ remains valid. The growth of these semimagnetic crystals with such a high concentration of carriers proved to be a challenging technological task.

Currently, the most actively studied group of ferromagnetic semiconducting materials are III-V semiconductors with $\mathrm{Mn}$, namely $\mathrm{Ga}_{1-x} \mathrm{Mn}_{x} \mathrm{As}$ and $\mathrm{In}_{1-x} \mathrm{Mn}_{x}$ As. Since the equilibrium solubility limit of $\mathrm{Mn}$ in III-V semiconductors is very low (at doping level) the ferromagnetic properties are observed only in thin layers grown by nonequilibrium method of low temperature molecular beam epitaxy (LT MBE) $[14,15]$. Although the record reported Curie temperature in GaMnAs is $110 \mathrm{~K}$, the well documented value is about $70 \mathrm{~K}$ for $\mathrm{Ga}_{1-x} \mathrm{Mn}_{x} \mathrm{As}$ with $x \approx 0.05$ and conducting hole concentration $p \approx 2 \times 10^{20} \mathrm{~cm}^{-3}$. In contrast to IV-VI and II-VI semiconductors, Mn forms in GaMnAs and InMnAs an elec- 
trically active acceptor center and is responsible for the high $p$-type conductivity observed in these layers. This center can be viewed as $\mathrm{Mn}^{2+}$ ion plus a weakly coupled hole. Electron paramagnetic resonance studies indicate that the simplest possible candidate, i.e. $\mathrm{Mn}^{3+}$ center observed in bulk crystals of some III-V materials, is not encountered in LT MBE epitaxial layers [16]. For the description of ferromagnetism in III-V semiconductors with Mn the models based on the RKKY interaction are usually invoked [14]. Recently, a new quantitative mean field approach based on the Zener model was proposed to explain ferromagnetism of III-V and II-VI compound semiconductors and group IV elemental semiconductors [17]. It indicates the importance of wide-gap semiconductor materials such as, e.g., $\mathrm{Ga}_{1-x} \mathrm{Mn}_{x} \mathrm{~N}$ as a possible candidate for semiconductor materials ferromagnetic at room temperature.

In II-VI semimagnetic semiconductors the interspin exchange interactions are dominated by antiferromagnetic superexchange mechanism. However, the theoretical analysis indicates that these materials might show ferromagnetic properties for high enough $p$-type doping. The basic mechanism is the compensation of antiferromagnetic superexchange interaction by ferromagnetic RKKY contribution [18]. Although $\mathrm{Mn}$ forms in II-VI materials a regular electrically neutral $\mathrm{Mn}^{2+}$ center, it tends to interfere with the $p$-type doping, e.g., by strongly compensating $\mathrm{P}$ acceptors in $\mathrm{ZnMnTe}$ with $x>0.03$. The conditions necessary for the formation of the ferromagnetic phase in II-VI semiconductors with Mn (hole concentration of $10^{20} \mathrm{~cm}^{-3}$ and Mn content of about (3-5)\%) were recently realized in MBE epitaxial layers of ZnMnTe doped with $N$ [19] and bulk ZnMnTe crystals doped with $\mathrm{P}[20]$. The dominance of ferromagnetic interactions were indeed experimentally observed in both materials. It was also shown that the band structure effects related to the lower dimensionality of the system might be beneficial for the formation of a ferromagnetic state as observed in CdMnTe-CdMgZnTe:N modulation doped quantum wells [21].

\section{Ferromagnetic ultrathin semiconductor layers}

Semiconductor ferromagnetic structures are usually composed of very thin (1-10 nm) ferromagnetic and nonmagnetic layers organized in the form of superlattice, trilayer, heterostructure or other planar multilayers (see Fig. 1). For such a thin ferromagnetic layer the new important effects must be considered, among others, the effect of stress induced by the lattice- or thermal-mismatch between the magnetic and nonmagnetic layers and the substrate, and the effect of strongly reduced thickness of magnetic layer on its basic magnetic properties such as the Curie temperature and magnetic anisotropy constant. Both these factors may dramatically change the magnetic properties of an ultrathin layer of a ferromagnet as compared to its bulk crystal form. For example, in the case of ultrathin ferromagnetic layer the magnetic system becomes effectively two-dimensional and the very existence of a ferromagnetic state may be questioned in view of the Mermin-Wagner theorem [22]. This theorem states that the ferromagnetic order cannot be sustained in the 2D system with ferromagnetic isotropic short-range exchange interactions. We will discuss these problems for the case of EuS-PbS semiconductor ferromagnetic multilayers. 
(a)

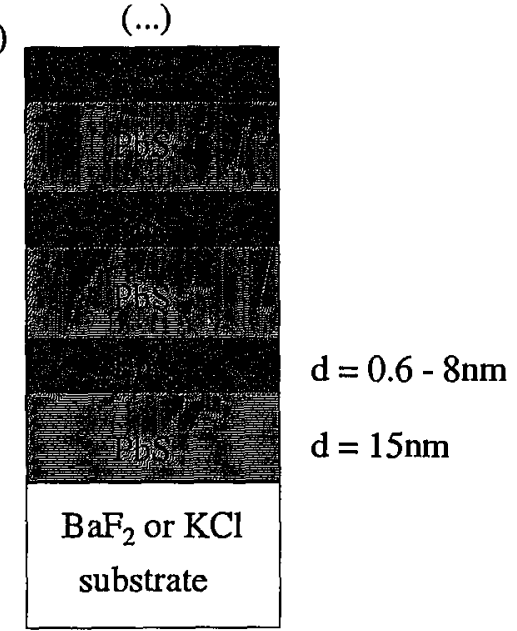

(b)

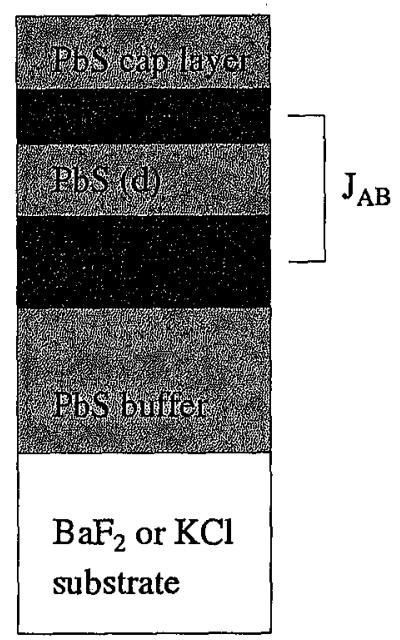

Fig. 1. The scheme of semiconductor ferromagnetic superlattice (a) and asymmetric trilayer (b) for the case of EuS-PbS system. The indicated thicknesses of layers correspond to the structures studied in Sec. 2 and Sec. 3.

A model ferromagnetic-nonmagnetic multilayer system discussed in here is EuS intercalated with $\mathrm{PbS}$. EuS and $\mathrm{PbS}$ crystallize in the same cubic (rock salt) crystal structure and the lattice mismatch between these two crystals is only $0.5 \%$. Since $\mathrm{PbS}$ is a typical diamagnet, magnetically the EuS-PbS multilayers are all-semiconducting ferromagnet-diamagnet nanostructures [23]. EuS-PbS multilayers form $\mathrm{PbS}$ multiple quantum wells with fundamental electronic transitions in the infrared $[24,25]$. We studied two series of EuS-PbS multilayer samples with a similar spectrum of layer thickness but with different layer orientation and different substrates. The EuS layer thickness ranged from 2 to 24 monolayers (ML), while the $\mathrm{PbS}$ layers were kept relatively thick $\left(d_{\mathrm{PbS}} \approx 50 \mathrm{ML}\right)$ to assure the lack of any magnetic coupling between the ferromagnetic layers. A few thick EuS-PbS bilayers $\left(d_{\mathrm{Eus}} \approx 200 \mathrm{ML}\right)$ were grown on $\mathrm{KCl}(100)$ and $\mathrm{BaF}_{2}(111)$ as a reference to bulk EuS crystals.

In Fig. 2a we present the dependence of the Curie temperature of EuS-PbS multilayers on the thickness of EuS layer. The ferromagnetic transition temperatures of the EuS-PbS structures on $\mathrm{BaF}_{2}$ (111) are shifted to lower temperatures by about $4 \mathrm{~K}$ with respect to the structures grown on $\mathrm{KCl}(100)$. This effect is attributed to the thermal stress in the EuS-PbS system as will be discussed below. For structures with EuS layers thinner than roughly $10 \mathrm{ML}$, the ferromagnetic Curie temperature $T_{\mathrm{C}}$ decreases substantially with decreasing thickness of EuS. In a simple physical picture this effect originates from the reduction of the number of magnetic neighbors of magnetic ions located close to the interfaces (see Fig. 2b). In the mean field model $T_{\mathrm{C}}$ is determined by the energy of the ground state of a ferromagnet: $k_{\mathrm{B}} T_{\mathrm{C}}=\frac{2}{3} S(S+1)\left(z_{1} J_{1}+z_{2} J_{2}\right)$, and scales by the average number of magnetic neighbors $\left(\bar{z}_{i}\right)$. For EuS, the magnetic interactions are important only for nearest $\left(z_{1}\right)$ and next nearest $\left(z_{2}\right)$ magnetic neighbors. Since the number of 
magnetic neighbors varies for ions located in different layers, the average number of neighbors depends on the number $n$ of EuS monolayers in the layer, as well as on the growth direction, and on the intermixing profile at the interface [23]. The final expression for the Curie temperature is $T_{\mathrm{C}}{ }^{(n)}=T_{\mathrm{C}}^{(\mathrm{b})}(1-c / n)$, where $c$ is a numerical parameter [23]. The application of other concepts (finite size scaling or exact calculations for the Ising systems) to the analysis of the thickness dependence of the Curie temperature in EuS-PbS multilayers is discussed in [23].

(a)

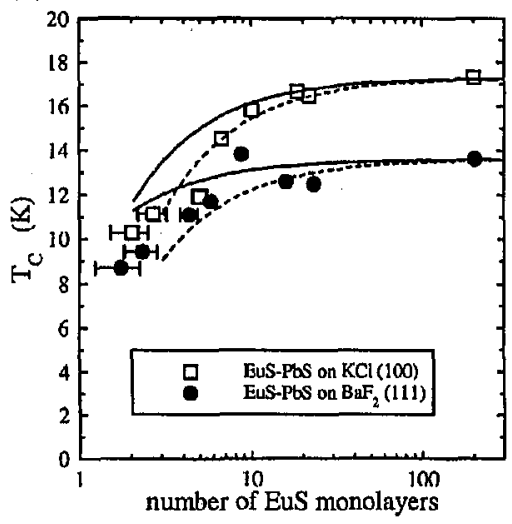

(b)
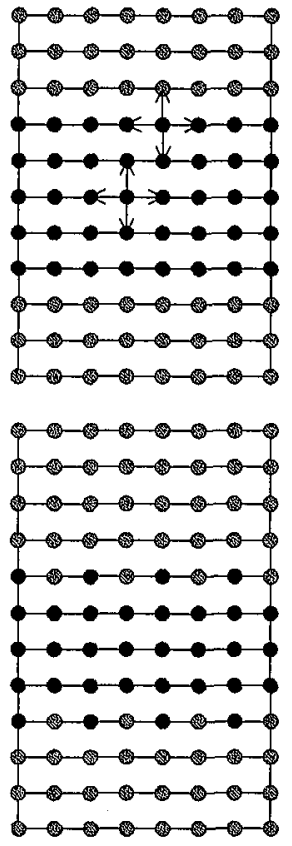

sharp interface

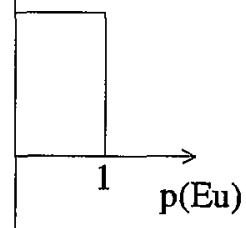

diffused interface

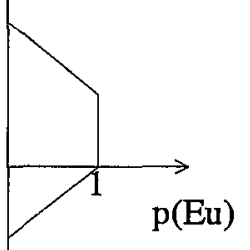

Fig. 2. (a) The thickness dependence of the Curie temperature $T_{\mathrm{C}}$ for two series of EuS-PbS multilayers grown on $\mathrm{KCl}(100)$ substrate (open squares), and on $\mathrm{BaF}_{2}$ (111) substrate (full circles). Solid lines correspond to the mean field calculations for the case of sharp EuS-PbS interface, whereas the dashed lines correspond to the case of intermixed interface of $2 \mathrm{ML}$ width. Part (b) illustrates the role of the, so-called, missing magnetic bonds of magnetic ions located close to the interfaces. Full dots represent magnetic ions $(\mathrm{Eu})$.

The Curie temperatures $T_{\mathrm{C}}$ of EuS-PbS multilayers are shifted with respect to the Curie temperature of bulk EuS crystal $T_{\mathrm{C}}^{(\mathrm{b})}$, with the shift $\Delta T_{\mathrm{C}}=$ $T_{\mathrm{C}}-T_{\mathrm{C}}^{(\mathrm{b})}$ depending on the substrate. For thick EuS-PbS bilayers grown on $\mathrm{KCl}(100)$ substrate, $T_{\mathrm{C}}$ is about $1 \mathrm{~K}$ higher, whereas for EuS-PbS bilayers grown on $\mathrm{BaF}_{2}(111), T_{\mathrm{C}}$ is about $3 \mathrm{~K}$ lower compared to bulk crystals for which $T_{\mathrm{C}}=16.6 \mathrm{~K}$ (Fig. 2a). We attribute this shift of ferromagnetic critical temperature to the in-plane biaxial stress present in EuS-PbS structures. This stress changes the interspin distances and the exchange integrals $J_{i}$, eventually changing the ferromagnetic transition temperature [23]. An analysis shows that the most 
important source of stress in EuS-PbS multilayers is the thermal stress due to the difference between the thermal expansion coefficients (TEC) of the substrate and the multilayer. The effect of TEC-stress was calculated for the temperature interval from the growth temperature $T_{\mathrm{g}}=520 \mathrm{~K}$ down to $T_{\mathrm{C}}=10 \mathrm{~K}$ for EuS-PbS bilayers grown on $\mathrm{KCl}$ and on $\mathrm{BaF}_{2}$ using a model assuming that: (1) at the growth temperature $T_{\mathrm{g}}$ the stress due to the lattice mismatch between substrate and PbS-buffer is fully relaxed, and (2) all the TEC-stress builds in the structure when the sample is cooled down. Using the recent neutron scattering data for the lattice parameter dependence of the Curie temperature of EuS [26], and the calculated changes of the in-plane and normal to the plane lattice parameters induced by TEC-stress, we estimated the shift $\Delta T_{\mathrm{C}}$ of the critical temperature of stressed EuS-PbS structure. Our calculations indicate that $T_{\mathrm{C}}$ of TEC-strained EuS-PbS/KCl could be shifted by $4.5 \mathrm{~K}$ higher with respect to the $T_{\mathrm{C}}$ of the free standing EuS-PbS structure. We have observed this effect experimentally by studying the temperature dependence of the ac magnetic susceptibility $\chi(T)$ of EuS-PbS structure on the $\mathrm{KCl}$ substrate and then the $\chi(T)$ dependence for the same structure without the substrate (i.e. free standing EuS-PbS structure with $\mathrm{KCl}$ substrate removed by dissolving in water) [23]. For the measurements, the free-standing multilayer is located on glass to which it is attached by weak van der Waals forces with negligible transmission of stress form the supporting glass. In Fig. 3a one can see that the $\chi(T)$ curves are shifted by about $3 \mathrm{~K}$ in a way expected for the action of the TEC-stress. In the case of EuS-PbS on the $\mathrm{BaF}_{2}$ (111) substrate the effect of thermal stress is one order of magnitude smaller due to much smaller difference between TEC of $\mathrm{BaF}_{2}$ and the multilayer. Recently, the same effect of stress on the Curie temperature was observed in related EuS-PbSe multilayers [27].

(a)

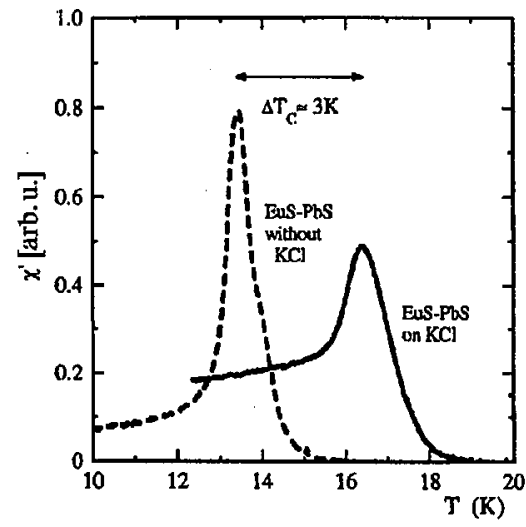

(b)

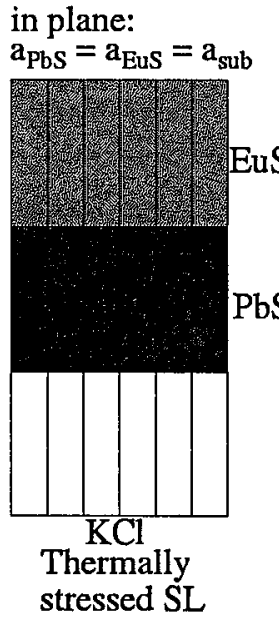

in plane:
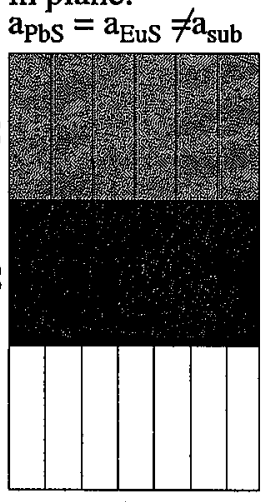

glass

Free standing SL

Fig. 3. (a) The temperature dependence of ac magnetic susceptibility for [EuS(18 ML)-PbS(59 ML)] multilayer on $\mathrm{KCl}(100)$ substrate, and for the same layer without the substrate. Part (b) illustrates the effect of thermal stress responsible for the shift of the $\chi(T)$ dependence presented in (a). 


\section{Interlayer exchange in ferromagnetic semiconductor superlattices}

The phenomenon of interlayer exchange coupling i.e. the interaction between two metallic ferromagnetic layers separated by a nonmagnetic metal layer was discovered in the late 1980's. Since then, it proved to be a rich field of fundamental research in modern magnetism and played key roles in many technological applications. So far, the vast scientific activity in this field has been focused on metallic systems in which the interlayer coupling can be explained in terms of quantum interference of electron waves of quasi-free conducting carriers in spin dependent potential of magnetic multilayer or, equivalently, via the spin polarization of conducting carriers (an analog of the RKKY interaction $[2,3]$ ). The necessary condition for the RKKY mechanism to play a significant role is the presence of a high concentration of carriers in the multilayer. The extension of this field to entirely nonmetallic systems based on semiconductors and insulators is motivated by two important factors: (1) the basic question as to whether interlayer coupling can exist in systems with negligibly small concentration of conducting carriers, and (2) the applicational idea of ferromagnetic elements epitaxially incorporated into the semiconductor electronic circuits.

Theoretical models developed for semiconductor ferromagnetic multilayers suggest that the interlayer coupling is due to the $s p-d$ exchange via carriers localized on shallow donor centers in the nonmagnetic spacer [28, 29], or by spin polarization of valence band electrons brought about by virtual electronic transitions over the energy gap of a semiconductor (an analog of the Bloembergen-Rowland mechanism) [30]. Another theoretical approach, based on the tight-binding method, has also been proposed to find the interlayer coupling strength by calculating the energy difference between the parallel and antiparallel spin configurations in successive magnetic layers [31]. Finally, the dipolar interactions can also be important in the case of ferromagnetic systems with significant interface roughness or in-plane domain structure [32]. For the ideal ferromagnetic planar system this mechanism is negligibly small.

The main experimental methods which can be employed to study the interlayer exchange in ferromagnetic multilayers are based on magnetization, ferromagnetic resonance, Brillouin light scattering, and neutron diffraction measurements.

The measurements of magnetic field dependence of magnetization $(M(H)$ loop) are usually performed by superconducting quantum interference device (SQUID) or magnetooptically by the Kerr effect measurements. This method provides a direct way to detect antiferromagnetically coupled layers (see Fig. 4) but is not conclusive for the case of ferromagnetic coupling. In the latter case one should use asymmetric trilayer structures (see Fig. 1b) to allow for the clear distinction between the non-coupled layers due to their different coercive fields (the method used, e.g., in the studies of GaMnAs-AlGaAs trilayers [33]).

In ferromagnetic resonance (FMR) measurements the interlayer exchange coupling is detected as the two-mode behavior of the system. Apart from the regular, so called, acoustic FMR mode (corresponding to the in-phase precession of magnetic moments of ferromagnetic layers), the optical mode is also observed (related to the out-of-phase precession of magnetic moments of the coupled layers). 

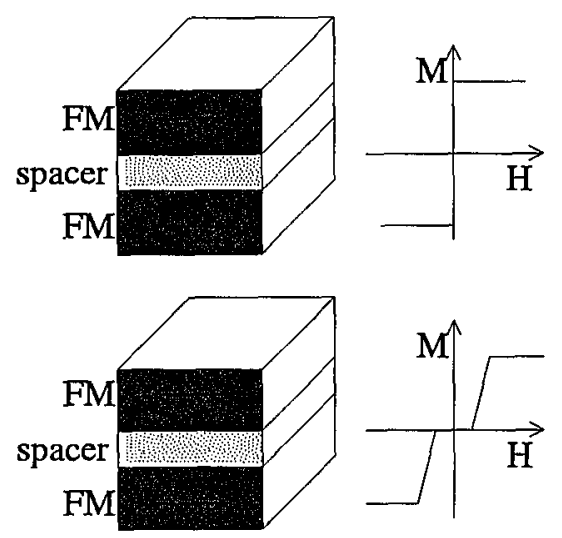

Fig. 4. The scheme illustrating the influence of the interlayer exchange interaction on the $M(H)$ dependence in magnetic trilayers: upper panel - ferromagnetically coupled layers; lower panel - antiferromagnetically coupled layers.

In principle, the position of FMR lines carries the information on both the sign and the strength of the interlayer coupling. However, the important drawback of this method is related to the zero intensity of the optical FMR mode for symmetric ferromagnetic trilayers [1]. It requires the use of asymmetric trilayer structures (Fig. 1b) with different FMR resonant fields due to different magnetic anisotropies. For the quantitative determination of the strength of the interlayer exchange interaction one has to compare FMR spectra for a series of identical trilayers differing only with respect to the thickness of the nonmagnetic spacer. This method was applied to EuS-PbS trilayers [34].

The third method used for the study of interlayer exchange in semiconductor ferromagnetic multilayers is neutron diffraction technique. This powerful technique proved to be very efficient in the analysis of both ferromagnetically and antiferromagnetically coupled EuS-PbS superlattices. In this method the interlayer coupling is detected by the observation of the correlations between the direction of the magnetization vectors of consecutive ferromagnetic layers in the multilayer. The evidence is particularly strong for the antiferromagnetically coupled multilayers when the new magnetic periodicity (twice bigger than the chemical superlattice periodicity) leads to the new set of diffraction peaks.

In all-semiconductor systems the interlayer exchange interaction was experimentally observed only in semiconductor antiferromagnetic-diamagnetic EuTe$\mathrm{PbTe}, \mathrm{MnTe}-\mathrm{ZnTe}$ and $\mathrm{MnTe}-\mathrm{CdTe}$ superlattices [35-37]. The experimental conclusion was based entirely on neutron diffraction studies. For ferromagnetic-nonmagnetic semiconductor systems, till recently, the only report concerned ferromagnetic interlayer exchange detected in magnetic hysteresis measurements in $\mathrm{Ga}_{1-x} \mathrm{Mn}_{x} \mathrm{As}-\mathrm{AlGaAs}$ asymmetric trilayers with two GaMnAs layers with $x=0.02$ and $x=0.04$ and different coercive fields [35]. The coupling is believed to be mediated by holes in the nonmagnetic AlGaAs layer i.e. by the RKKY mechanism known in metallic systems. 
For ferromagnetic semiconductor multilayers both recent theoretical papers (discussed above) and a very limited experimental data indicated only ferromagnetic interlayer coupling. Since it is an antiferromagnetic interlayer coupling which is vital for the operation of most of important magnetic thin layer structures such as, e.g., spin valves, it is important to find ferromagnetic semiconductor structures with antiferromagnetic interlayer exchange.

Recently, the antiferromagnetic interlayer exchange was discovered in EuS-PbS/KCl(001) multilayers with $\mathrm{PbS}$ thickness of about $1 \mathrm{~nm}$. The coupling is observed both in neutron diffraction and magnetization measurements performed for superlattices, symmetric trilayers and asymmetric trilayers [38]. It was also proposed that the coupling may be driven by a new mechanism related to the band structure effects sensitive to the magnetic structure of the multilayer [31, 38].

\section{Summary}

We have briefly discussed recent progress in the field of semiconductor ferromagnetic structures. These new magnetic low dimensional materials offer a qualitatively new extension to the well known field of magnetic metallic multilayers. The discussion of the properties related to dimensionality effects and stress effects was presented for the case of model Heisenberg ferromagnetic-nonmagnetic multilayer EuS-PbS. We have also analyzed recent theoretical and experimental efforts aimed at the understanding of the phenomenon of interlayer exchange coupling in semiconducting systems with negligible carrier concentration. The discovery of antiferromagnetic interlayer exchange in EuS-PbS (100) multilayers opens unique possibilities for growing new structures such as, e.g., EuS-PbS-EuS-EuTe all-semiconductor spin valves.

\section{Acknowledgments}

This work was partially supported by the Committee for Scientific Research project No. 2 P03B 15418.

\section{References}

[1] Ultrathin Magnetic Structures, Eds. J.A.C. Bland, B. Heinrich, Springer-Verlag, Berlin 1994.

[2] P. Bruno, Phys. Rev. B 52, 411 (1995).

[3] A. Fert, P. Grünberg, A. Barthelemy, P. Petroff, W. Zinn, J. Magn. Magn. Mater. 140-144, 1 (1995).

[4] G.A. Prinz, Science 282, 1660 (1998).

[5] M. Johnson, R. Silsbee, Phys. Rev. B 35, 4959 (1987).

[6] G.J. Strijkers, J.T. Kohlhepp, H.J.M. Swagten, W.J.M. de Jonge, Phys. Rev. Lett. 84, $1812(2000)$.

[7] S. Methfessel, D.C. Mattis, Magnetic Semiconductors, Springer, Berlin 1968. 
[8] E.L. Nagaev, Physics of Magnetic Semiconductors, Mir, Moscow 1983.

[9] A. Mauger, C. Godart, Phys. Rep. 141, 51 (1986).

[10] T. Story, G. Karczewski, L. Świerkowski, R.R. Gałązka, Phys. Rev. B 42, 10477 (1990).

[11] W.J.M. de Jonge, H.J.M. Swagten, J. Magn. Magn. Mater. 100, 322 (1991).

[12] P.J.T. Eggenkamp, H.J.M. Swagten, T. Story, V.I. Litvinov, C.H.W. Swüste, W.J.M. de Jonge, Phys. Rev. B 51, 15250 (1995).

[13] P. Eazarczyk, T. Story, M. Arciszewska, R.R. Gałązka, J. Magn. Magn. Mater. 169, 151 (1997).

[14] H. Ohno, Science 281, 951 (1998).

[15] H. Ohno, Acta Phys. Pol. A 94, 155 (1998).

[16] J. Szczytko, W. Mac, A. Stachow, A. Twardowski, P. Becla, J. Tworzydło, Solid State Commun. 99, 927 (1996).

[17] T. Dietl, H. Ohno, F. Matsukura, J. Cibert, D. Ferrand, Science 287, 1019 (2000).

[18] A. Haury, A. Wasiela, A. Arnoult, J. Cibert, S. Tatarenko, T. Dietl, Y. Merle d'Aubigné, Phys. Rev. Lett. 79, 511 (1977).

[19] D. Ferrand, J. Cibert, A. Wasiela, C. Bourgognon, S. Tatarenko, G. Fishman, S. Koleśnik, J. Jaroszyński, T. Dietl, B. Barbara, D. Dufeu, J. Appl. Phys. 87, 6451 (2000).

[20] Le Van Khoi, unpublished.

[21] T. Dietl, A. Haury, Y. Merle d'Aubigné, Phys. Rev. B 55, 1 (1997).

[22] Magnetic Properties of Layered Transition Metal Compounds, Ed. L.J. de Jongh, Kluwer Academic, Dordrecht 1990.

[23] A. Stachow-Wójcik, T. Story, W. Dobrowolski, M. Arciszewska, R.R. Gałązka, M.W. Kreijveld, C.H.W. Swuste, H.J.M. Swagten, W.J.M. de Jonge, A. Twardowski, A.Yu. Sipatov, Phys. Rev. B 60, 15220 (1999).

[24] I.V. Kolesnikov, V.A. Litvinov, A.Yu. Sipatov, A.I. Fedorenko, A.E. Yunovich, Sov. Phys.-JETP 67, 1431 (1988).

[25] L. Kowalczyk, J. Sadowski, R.R. Gałązka, A. Stachow-Wójcik, A.Yu. Sipatov, V.V. Volobuev, V.A. Smirnov, V.K. Dugaev, Acta Phys. Pol. A 94, 397 (1998).

[26] I.N. Goncharenko, I. Mirebeau, Phys. Rev. Lett. 80, 1082 (1998).

[27] T. Story, M. Arciszewska, M. Czernyshowa, W. Dobrowolski, W. Mac, A. Twardowski, R. Świrkowicz, A.Yu. Sipatov, to be published.

[28] P. Shevchenko, L. Świerkowski, J. Oitmaa, J. Magn. Magn. Mater. 177-181, 1168 (1998).

[29] T.M. Rusin, Phys. Rev. B 58, 2107 (1998).

[30] V.K. Dugaev, V.I. Litvinov, W. Dobrowolski, T. Story, Solid State Commun. 110, 351 (1999).

[31] J. Blinowski, P. Kacman, Acta. Phys. Pol. A 92, 719 (1997).

[32] J.A. Borchers, J.A. Dura, J. Unguris, D. Tulchinsky, M.H. Kelley, C.F. Majkrzak, S.Y. Hsu, R. Loloee, W.P. Pratt, Jr., J. Bass, Phys. Rev. Lett. 82, 2796 (1999).

[33] N. Akiba, F. Matsukura, A. Shen, Y. Ohno, H. Ohno, A. Oiwa, S. Katsumoto, Y. Iye, Appl. Phys. Lett. 73, 2122 (1998). 
[34] T. Story, C.H.W. Swüste, H.J.M. Swagten, W.J.M. de Jonge, A. Stachow-Wójcik, M. Arciszewska, A.Yu. Sipatov, in: Conference Booklet of XXVIII Int. School Phys. Semicond. Compounds "Jaszowiec '99", Ed. W. Szuszkiewicz, Institute of Physics, Warsaw 1999, p. 128.

[35] H. Kepa, K.I. Goldman, T.M. Giebułtowicz, C.F. Majkrzak, G. Springholz, H. Krenn, S. Holl, F. Schinagl, G. Bauer, Physica E 2, 399 (1998).

[36] J.J. Rhyne, J. Lin, J.K. Furdyna, T.M. Giebułtowicz, J. Magn. Magn. Mater. 177-181, 1195 (1998).

[37] V. Nunez, T.M. Giebułtowicz, W. Faschinger, G. Bauer, H. Sitter, J.K. Furdyna, J. Magn. Magn. Mater. 140-144, 633 (1995).

[38] H. Kępa, J. Kutner-Pielaszek, J. Blinowski, A. Twardowski, C.F. Majkrzak, T. Story, P. Kacman, R.R. Gałazka, K. Ha, H.J.M. Swagten, W.J.M. de Jonge, A.Yu. Sipatov, T.M. Giebultowicz, submitted to Phys. Rev. Lett. 